\title{
Contribuição à taxonomia de Marlierea (Myrciinae; Myrtaceae) no Brasil'
} Contribution to the taxonomy of Marlierea (Myrciinae; Myrtaceae) in Brazil

\author{
Alessandro S. do Rosário ${ }^{2,5}$, José Fernando A. Baumgratz ${ }^{3}$ \& Ricardo de S. Secco ${ }^{4}$
}

\begin{abstract}
Resumo
Apresenta-se uma circunscrição taxonômica de Marlierea e três novos registros de ocorrência para o gênero na Amazônia brasileira, sendo dois para o Brasil, três para o estado do Pará e um para o estado do Amazonas. Comentários sobre características diagnósticas e afinidades taxonômicas de cada espécie, bem como os respectivos dados de distribuição geográfica e ambientes típicos de ocorrência são também fornecidos. Recomendações para atualização da lista de espécies de Myrtaceae da flora do Brasil também são apresentadas. Palavras-chave: Amazônia, circunscrição taxonômica, fitofisionomia, morfologia, novos registros.
\end{abstract}

\begin{abstract}
This paper presents a taxonomic circumscription of Marlierea and three new records of occurrence for the genus in the Brazilian Amazon, two in Brazil, three for the State of Para and one for the State of Amazonas. Comments on the morphology and affinity of taxa are provided to facilitate recognition of each species, as well as their geographical distribution data and typical environments occurrence of these species in the Amazon region. Recommendations for updating the list of Brazilian Myrtaceae species are presented.
\end{abstract}

Key words: Amazon, morphology, new records, phytophysiognomy, taxonomic circumscription.

\section{Introdução}

Marlierea Cambess. está representado por 62 espécies no Brasil, sendo 12 na Amazônia brasileira (Sobral et al. 2012), porém, Rosário (2012) atualizou este número para 14 espécies amazônicas. Apresenta distribuição exclusivamente Neotropical e está entre os menores, mais complexos e menos estudados gêneros de Myrtaceae (Rosário \& Secco 2006). McVaugh (1968) relata que Marlierea reúne mais de 90 espécies, estando mais de 50\% concentrados no sul do Brasil, 25\% na região das Guianas e o restante nas Antilhas ou em outra parte da América do Sul tropical.

Esse gênero distingue-se basicamente dos demais que compõem a subtribo Myrciinae sensu Berg (1855-56, 1857-59, denominada Myrcioideae) e Rosário (2012), pela deiscência do cálice e exposição da corola no botão floral, como segue: cálice com deiscência valvar ou quincuncial e corola indeiscente, porém exposta no botão floral adulto, em Myrcia DC. ex Guill. e Gomidesia O.Berg; e cálice indeiscente e corola inclusa no botão floral adulto, em Calyptranthes Sw. e Marlierea (Rosário 2012). Neste último caso, observa-se uma única peça floral de revestimento sobre o androceu e gineceu, sem haver distinção entre o cálice e a corola durante o processo de antese. Portanto, a deiscência do cálice torna-se importante para a distinção desses táxons, sendo circuncisa em Calyptranthes, formando uma caliptra, caduca ou não, e em Marlierea, parcial ou totalmente irregular, formando sépalas de tamanhos e formas irregulares entre si (McVaugh 1958; Landrum \& Kawasaki 1997; Rosário 2012).

A estreita afinidade taxonômica, a fragilidade das circunscrições e a dificuldade de identificar esses quatro gêneros têm influenciado vários autores a considerarem artificial a classificação desses táxons e proporem a sinonimização de alguns deles ou mesmo considerá-los um único táxon

\footnotetext{
' Parte da tese de Doutorado do primeiro autor.

${ }^{2}$ Museu Paraense Emílio Goeldi, Coord. Botânica, Av. Perimetral 1901, 66040-170, Terra Firme, Belém, PA, Brasil.

${ }^{3}$ Instituto de Pesquisas Jardim Botânico do Rio de Janeiro, DIPEQ, R. Pacheco Leão 915, 22460-030, Jardim Botânico, Rio de Janeiro, RJ, Brasil.

${ }^{4}$ Museu Paraense Emílio Goeldi, Coord. Botânica, Av. Perimetral 1901, 66040-170, Terra Firme, Belém, PA, Brasil.

${ }^{5}$ Autor para correspondência: asrosario@museu-goeldi.br
} 
(McVaugh 1968; Kawasaki \& Holst 1994; Landrum \& Kawasaki 1997; Holst et al. 2003; Sobral 2003; Lucas et al. 2005, 2007, 2011; Wilson 2011).

Por outro lado, há ainda pontos taxonômicos conflitantes relativos às Myrciinae e que precisam ser resolvidos futuramente: (a) a designação do único gênero, pois tem sido proposto "Myrcia s.l.", apesar de o nome "Calyptranthes" ter prioridade sobre os demais (decisão a ser tomada no próximo Congresso Internacional de Botânica, em 2017, na China); (b) a não formalização dos clados como categorias taxonômicas infra-genéricas para o único gênero a ser considerado; e (c) a necessidade de serem incluídas espécies amazônicas em análises filogenéticas moleculares, as quais praticamente se restringem às espécies do sul do continente sulamericano e incluem pouquíssimas espécies de ampla distribuição na Região Neotropical.

Nesse contexto, tanto estudos florísticos quanto taxonômicos sobre as Myrciinae amazônicas contribuem com novas informações sobre caracteres morfológicos diagnósticos, em nível genérico e específico, e até mesmo informativos para análises filogenéticas, principalmente considerando-se novas proposições sistemáticas para a família (Rosário 2012). Esses estudos também permitem reavaliar taxonomicamente Marlierea, fornecendo dados atualizados sobre a diversidade, distribuição geográfica, endemismos e especificidades de habitats, além de reconhecer padrões de distribuição geográfica e subsidiar com novas informações a lista das espécies de Myrtaceae da flora do Brasil (Sobral et al. 2012).

Considerando os recentes resultados obtidos por Rosário (2012), é apresentada uma circunscrição morfológica diagnóstica de Marlierea e três novos registros de ocorrência para o gênero na Amazônia brasileira, sendo dois para o Brasil, três para o estado do Pará e um para o estado do Amazonas. Apresentamse também comentários sobre circunscrições e afinidades taxonômicas, dados de distribuição geográfica e formações vegetacionais onde as espécies abordadas são encontradas no Brasil. Além disso, faz-se recomendações para a atualização da lista de espécies de Myrtaceae da flora do Brasil (Sobral et al. 2012), propondo-se a retirada de três espécies de Marlierea, das quais uma é aceita como sinônimo de Calyptranthes, bem como uma nova ocorrência para o Brasil (Amazônia brasileira).

\section{Material e Métodos}

O levantamento de coleções abrangeu principalmente os herbários da Região Norte do Brasil (EAFM, IAN, INPA, MG), além das coleções dos herbários R, RB, BHCB e UB, bem como fotografias de tipos nomenclaturais de Myrciinae depositados nos herbários BM, BR, F, K, MICH, M, MO, NY, P, US e U (acrônimos segundo Thiers continuously updated). As abreviações dos nomes dos autores dos táxons seguem Brummitt \& Powell (1992).

A identificação das espécies foi realizada com o auxílio de literatura especializada (McVaugh 1958, 1969; Holst et al. 2003; Rosário \& Secco 2006), bem como material identificado por especialistas, espécimes-tipo, fotos e/ou imagens digitalizadas e consultas a especialistas em Myrtaceae.

Analisou-se material procedente da área de estudo e, quando necessário, materiais adicionais foram consultados, além da elaboração de ilustrações e apresentação de dados fenológicos, distribuição geográfica e comentários sobre a distribuição nas fitofisionomias e afinidades taxonômicas. As análises morfológicas e mensurações em laboratório foram realizadas com o auxílio de microscópio estereoscópico CARL ZEISS $®$. Na caracterização morfológica foram utilizados os conceitos de McVaugh (1956, 1968, 1969), Radford et al. (1974) e de Barroso et al. (1999). A descrição do gênero está de acordo com Rosário (2012).

A caracterização das fitofisionomias foi baseada na classificação da vegetação proposta por Pires \& Prance (1985). Informações quanto ao período de floração (botões em pré-antese e totalmente abertos) e frutificação (ovários iniciando o desenvolvimento e frutos maduros prontos para a dispersão) das referidas espécies também foram obtidos em literatura e coleções de herbários.

Nas ilustrações, destacam-se os principais caracteres vegetativos e reprodutivos das espécies estudadas.

\section{Resultados e Discussão}

Marlierea Cambess. In: A.St.-Hil., Fl. bras. Merid. 2: 373. [folio ed. 269], tab. 156. 1829.

Árvores ou arbustos, monopodiais. Inflorescências geralmente em panículas, ou reduzidas à panículas de racemos (racemiformes), panículas de fascículos (fasciculiformes), axilares e/ou terminais; brácteas e bractéolas aciculadas a elíptico-ovais ou ausentes. Botão floral com sépalas 
totalmente soldadas entre si, apiculado ou não; cálice 4-5-mero após antese, deiscência parcial ou totalmente irregular, sépalas irregularmente denteadas ou arredondadas. Flores subsésseis ou nitidamente pediceladas; pétalas ausentes, raro 1-4(5); hipanto prolongado acima do ápice do ovário; zona do disco glabra ou pilosa; ovário $2(-3)$ locular, com 2 óvulos axilares por lóculo; embrião mircióide. Frutos bacáceos, globosos, raro costados, com cálice, estames e estilete geralmente persistentes.

\section{Novos registros de ocorrência para a Amazônia brasileira}

1. Marlierea bipennis (O.Berg) McVaugh, Mem. New York Bot. Gard. 10(1): 79. 1958. Fig. 1a-b

Marlierea bipennis é endêmica da Amazônia, conhecida anteriormente para a Guiana e Brasil (ocorrendo em floresta de igapó no estado do Amazonas) (Rosário \& Secco 2006). A coleta dessa espécie na Serra dos Carajás representa o primeiro registro para o estado do Pará, onde ocorre em floresta de terra firme e pode apresentar hábito lianescente (Rosário 2012). Tem distribuição restrita à Amazônia, com ocorrência também na Guiana e no Brasil, em floresta de igapó, no estado do Amazonas (Rosário \& Secco 2006).

Marlierea bipennis assemelha-se à $M$. ensiformis McVaugh por apresentar os ramos levemente comprimidos, com alas membranáceas em direção à porção distal. Porém, distingue-se pelas folhas com 5-10 cm compr. (vs. 13-15 cm compr.), coriáceas ( $v s$. cartáceas), as inflorescências em panículas axilares ( $v s$. panículas terminais, ocasionalmente axilares nos três nós distais dos ramos) e a raque cilíndrica a levemente comprimida ( $v s$. nitidamente comprimida).

Material examinado: BRASIL. PARÁ: Serra dos Carajás, Serra Norte, $5 \mathrm{~km}$ NE do campo da AMZA Exploration, ca. $6^{\circ} \mathrm{S}, 50^{\circ} 15^{\prime} \mathrm{W}, 15 . X .1977$, fr., C.C. Berg et al. 535 (INPA, MG, RB). AMAZONAS: NW of São Gabriel, near mouth of the rio Uaupés, 0005-08', 67¹0’W, VII.1979, fr., J.M. Poole 2085 (MG, MO).

Material adicional examinado: GUIANA. Cuyuni river, on the right bank below the Akaio Falls, 22.XI.1929, fl., N.Y. Sandwith 646 (RB).

2. Marlierea maguirei McVaugh, Mem. New York Bot. Gard. 10(1): 87. $1958 . \quad$ Fig. 1c-d

A coleta dessa espécie na Serra dos Carajás, no Pará, representa uma nova ocorrência para a Amazônia brasileira, e também, para o estado do Pará e Brasil. É encontrada em campos rupestres ferruginosos, conhecidos como vegetação de canga, a qual está associada a solos com alta concentração de minério de ferro. Holst et al. (2003) consideraram esta espécie como endêmica das regiões serranas da Venezuela.

Marlierea maguirei distingue-se facilmente das demais espécies do gênero por apresentar as estruturas vegetativas e reprodutivas glabras, pecíolo levemente alado, inflorescência com a raque curta (até $3 \mathrm{~cm}$ compr.) e botão floral apiculado, geralmente partido no ápice.

Material examinado: BRASIL. PARÁ: Marabá, Serra dos Carajás, N-3, 28.X.1985, bot., R. Secco \& O. Cardoso 642 (MG). Serra dos Carajás, N-3, 24.X.1992, fr., J. Sales \& C. Rosário 52 (MG).

\section{Marlierea schomburgkiana O.Berg, Linnaea} 29: 209. 1858.

Fig. 1e

Marlierea schomburgkiana é assinalada pela primeira vez para a Amazônia brasileira, o que representa uma nova ocorrência para o Brasil, bem como para os estados do Amazonas e Pará, onde tem distribuição restrita em floresta de terra firme no estado do Pará. Também é encontrada na Venezuela, Equador, Guiana, Suriname, Peru e Bolívia.

Similar a $M$. suaveolens Cambess. devido, principalmente, a aparente semelhança da forma do ápice das folhas, pois é abruptamente longo-caudado em M. schomburgkiana e longoacuminado em $M$. suaveolens. Diferencia-se, também, pela distribuição geográfica, pois é endêmica da Amazônia, enquanto M. suaveolens é restrita às regiões Sul e Sudeste do Brasil (Sobral et al. 2012). Segundo Holst (2002), as coleções provenientes do Peru apresentam pecíolos curtos e venação da lâmina foliar bem marcada, o que coincide com as características observadas nas coleções provenientes do Brasil (Amazonas e Pará). Vale ressaltar que nos espécimes coletados no Suriname e na Guiana, o pecíolo é mais longo, podendo chegar até $7 \mathrm{~mm}$ compr.

Berg (1857-1859) não menciona o coletor nem a localidade-tipo no protólogo de $M$. schomburgkiana, citando apenas "v. in herb. Berol.". Por esse motivo, a coleção Jenman 4176 (K, Univ. of Mich. neg 1000) foi escolhida por McVaugh (1958) como neótipo para a espécie, assinalando, posteriormente, que o exemplar-tipo tinha sido provavelmente destruído (McVaugh 1969).

As coleções Sandwith 613 e 922 (RB), provenientes da Guiana, estão representadas por dois ramos férteis, e compõem uma única exsicata, porém com duas etiquetas. Segundo os dados contidos nessas etiquetas a coleção Sandwith 613 


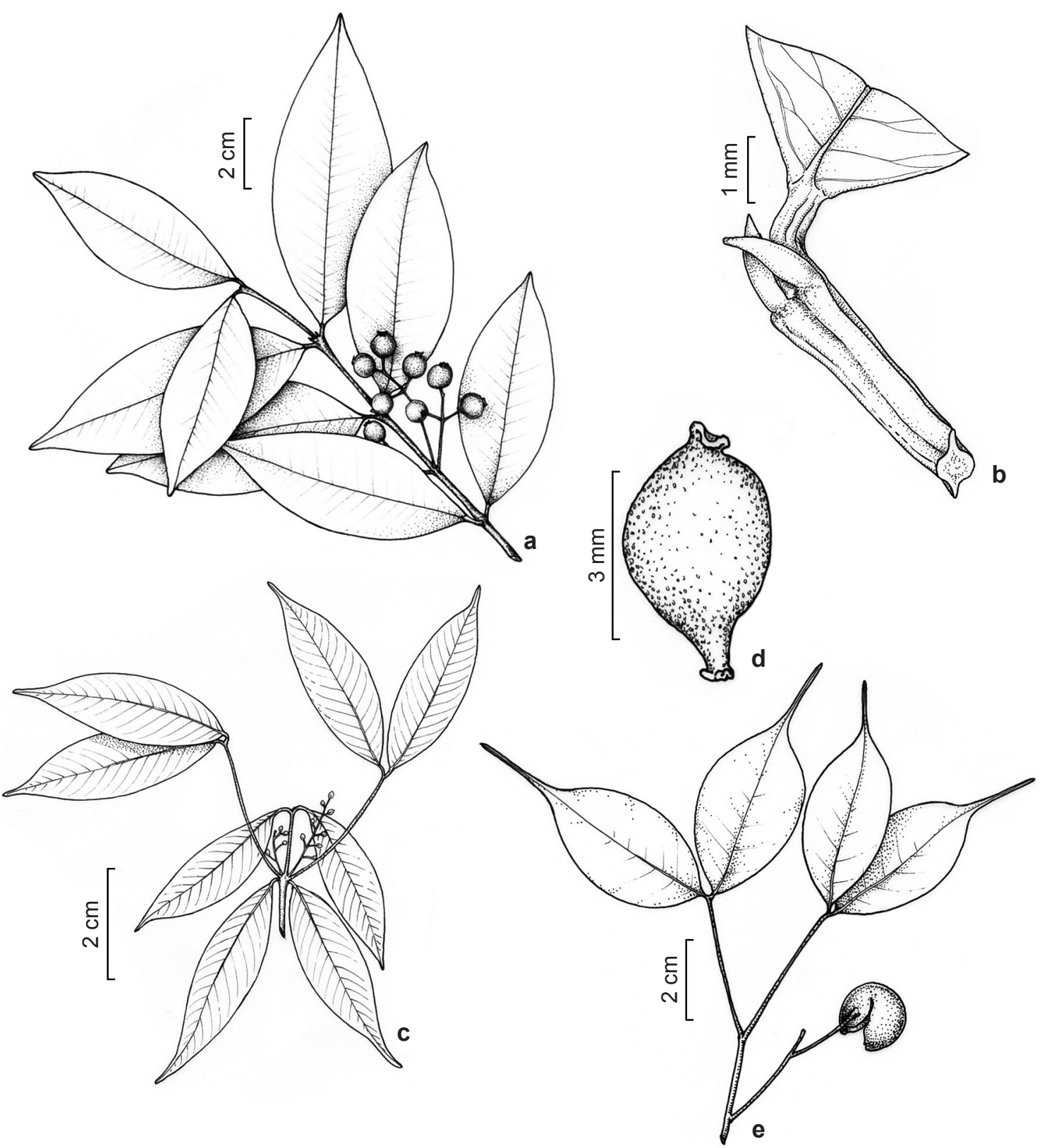

Figura 1 - a-b. Marlierea bipennis - a. ramo com frutos; b. ramo alado. c-d. Marlierea maguirei - c. ramo florífero; d. botão floral, evidenciando apículo partido. Marlierea schomburgkiana - e. ramo com fruto (a J.M. Poole 2085; b C.C. Berg 535; c-d R. Secco 642; e G.T. Prance 25536).

Figure 1 - a-b. Marlierea bipennis - a. branch with fruits; b. winged branch. c-d. Marlierea maguirei - c. branch in bloom; d. floral bud with the apiculous split at the apex. Marlierea schomburgkiana - e. branch with fruit (a J.M. Poole 2085; b C.C. Berg 535; c-d R. Secco 642; e G.T. Prance 25536).

foi coletada em novembro de 1929, enquanto Sandwith 922, em abril do mesmo ano. Depreendese que houve uma falha de transcrição de dados para a elaboração das respectivas etiquetas e essas amostras correspondem a indivíduos que ocorrem na mesma região. Apesar de o banco de dados JABOT (<www.jbrj.gov.br>), do Herbário RB, assinalar uma única data (18.X.1928) e um único número de coleta (613) para as duas coleções, aceitase, no momento, que ambos os espécimes foram 
coletados em 1929, uma vez que ainda não se teve acesso à caderneta de coletas de N.Y. Sandwith. Material examinado: BRASIL. PARÁ: BR 163, CuiabáSantarém, km 1234, 18.XI.1977, fr., G.T. Prance et at. 25536 (INPA, MG, NY). AMAZONAS: Manaus - Porto Velho, 15.III.1974, fr., G.T. Prance et al. 20553 (INPA, MG, NY). Material adicional examinado: SURINAME. Distr. Nickerie, 9.IX.1980, fr., J.C. Lindeman et al. 292 (BBS, MG, U). GUIANA. 1929, bot., N.Y. Sandwith 613, 922 (RB).

\section{Recomendações para a Lista de espécies da flora do Brasil}

4. Marlierea areolata McVaugh, Fieldiana, Bot. 29(3): 175. 1956.

Marlierea areolata foi excluída por Rosário (2012) do tratamento taxonômico do gênero para a Amazônia brasileira, uma vez que apenas um exemplar estéril esteve disponível para análise (Holst 8334, SEL), coletado no Acre. Segundo Holst (com. pessoal), a identificação que ele realizou deste espécime é duvidosa, uma vez que todas as amostras encontram-se estéreis. Por sua vez, Kawasaki \& Holst (2006) assinalam que esta espécie está entre as Myrtaceae endêmicas do Peru.

Desse modo, recomenda-se retirar $M$. areolata da lista de espécies de Myrtaceae da flora do Brasil, uma vez que se faz necessário realizar coletas de amostras férteis, a fim de que novos exemplares não só comprovem a ocorrência dessa espécie no território brasileiro, como corroborem a identificação correta da coleção supracitada.

5. Marlierea montana (Aubl.) Amshoff, Recueil Trav. Bot. Néerl. 39: 147. 1942.

Marlierea montana foi excluída do tratamento taxonômico do gênero para a Amazônia brasileira (Rosário 2012), uma vez que o único registro citado em banco de dados, como o JABOT (<www.jbrj. gov.br $>$ ), e correspondente ao exemplar Marinho 15 (RB), procedente do estado de Roraima, não foi localizado. Analisando uma imagem desse material disponível no sítio eletrônico do Herbário $\mathrm{RB}$, acredita-se que corresponda a um espécime de Myrcia subsessilis O.Berg, considerando principalmente as folhas subsésseis, oblongas, base obtusa a levemente cordada, ápice obtuso e nervuras intersecundárias nitidamente reticuladas. Esta espécie já é citada como ocorrente na Amazônia brasileira (Sobral et al. 2012).

Desse modo, recomenda-se retirar a citação de $M$. montana da lista de espécies de Myrtaceae da flora do Brasil para a Amazônia
(Sobral et al. 2012), uma vez que se faz necessário localizar e analisar de forma mais criteriosa o material supracitado.

Material examinado: BRASIL. RORAIMA: entre Boa Vista e Santa Helena, Rodovia BR-174. Quadrícula NB-20-ZD, 1.VII.1974, fl., L.R. Marinho 15 (RB, foto).

6. Marlierea uniflora McVaugh, Mem. New York Bot. Gard. 18(2): 69. 1969. (=Calyptranthes moaensis Alain).

Marlierea uniflora foi aceita como sinônimo de Calyptranthes moaensis Alain (Rosário 2012), considerando as sobreposições de características morfológicas, tanto vegetativas quanto florais, e principalmente pela deiscência caliptriforme do cálice. Estes autores comentam sobre essas características, corroborando as observações já assinaladas por Rosário (2012), que também analisou os exemplares-tipo de ambos os táxons. Neste trabalho, o autor também destaca que é necessário realizar novas coletas, visando ampliar o conhecimento sobre o referido táxon, pois o mesmo encontra-se muito mal representado em coleções de herbários.

Desse modo, recomenda-se retirar Marlierea uniflora da lista de espécies da flora e incluir Calyptranthes moaensis, que é um novo registro de ocorrência para o Brasil (Amazônia brasileira). Material examinado: BRASIL. AMAZONAS: São Gabriel da Cachoeira, rio Cubate, afluente do rio Içana, 4.XI.1987, f1., C. Farney 1886 (RB). São Gabriel da Cachoeira, entre as ilhas Açaí e Aparecida, rio Negro, 25.VII.1991, fl., G. Martinelli 14532 (RB).

\section{Agradecimentos}

Ao Instituto de Pesquisas Jardim Botânico do Rio de Janeiro, o apoio na execução do projeto com as Myrciinae no estado do Pará; ao Museu Paraense Emílio Goeldi, por disponibilizar a infraestrutura necessária para a realização dos estudos em Myrtaceae na Amazônia brasileira; à Fundação Amazônia Paraense de Amparo à Pesquisa a concessão de bolsa de doutorado, posteriormente substituída pelas bolsas DTI-1 e EV-B, do CNPq; e à Coordenação do projeto "Geoambientes, geodiversidade e valores de referência para metais pesados nas áreas de Canga Ferrífera em Carajás (PA), ICAAF 036/2011 (VALE/FAPESPA)". Ao ilustrador Carlos Alvarez, a confecção das pranchas. Ao Dr. Marcelo Souza e Dr. Marcos Sobral, as sugestões dadas durante o estudo taxonômico de Myrciinae. Ao CNPq, as Bolsas de Produtividade concedidas aos co-autores. 


\section{Referências}

Barroso, G.M.; Morim, M.P.; Peixoto, A.L. \& Ichaso, C.L.F. 1999. Frutos e sementes: morfologia aplicada à sistemática de dicotiledôneas. Ed. UFV, Viçosa. 443p.

Berg O.K. 1855-56. Revisio Myrtacearum Americae. Linnaea 27: 1-472.

Berg, O.K. 1857-1859. Myrtaceae. In: Martius, C.F.P. von.; Eichler, A. \& Urban, I. (eds.). Flora brasiliensis. Munchen, Wien, Leipzig, Vol. 14. Pp. 1-655.

Brummitt, R.K. \& Powell, C. 1992. Authors of plants names. Royal Botanical Garden, Kew. 732p.

Holst, B.K. 2002. New species and notes on Myrtaceae from northern South America. Selbyana 23: 137-180.

Holst, B.K.; Landrum, L. \& Grifo, F. 2003. Myrtaceae. In: Steyermark, J.A.; Berry, P.E.; Yatskievych, K. \& Holst, B.K. (eds.). Flora of the Venezuelan Guayana. Vol. 7. Missouri Botanical Garden Press, St. Louis. Pp. 1-99.

Kawasaki, M.L. \& Holst, B.K. 1994. New species and a new combinations in Myrtaceae from northeastern South America. Brittonia 46: 137-143.

Kawasaki, M.L. \& Holst, B.K. 2006. Myrtaceae endémicas del Perú. In: Leon, B. (ed.). El libro rojo de las plantas endémicas del Peru. Revista Peruana de Biología 13: 463-468.

Landrum, L. \& Kawasaki, M.L. 1997. The genera of Myrtaceae in Brazil: an illustrated synoptic treatment and identification keys. Brittonia 49: 508-536.

Lucas, E.J.; Belsham, S.R.; Nic Lughadha, E.M.; Orlovich, D.A.; Sakuragui, C.M.; Chase, M.W. \& Wilson, P.G. 2005. Phylogenetic patterns in the flesh-fruited Myrtaceae - preliminary molecular evidence. Plant Systematics and Evolution 251: 35-51.

Lucas, E.J.; Harris, S.A.; Mazine, F.F.; Belsham, S.R.; Nic Lughadha, E.M.; Telford, A.; Gasson, P.E. \& Chase, M.W. 2007. Suprageneric phylogenetics of Myrteae, the generically richest tribe in Myrtaceae (Myrtales). Taxon 56: 1105-1128.

Lucas, E.J.; Matsumoto, K.; Harris, S.A.; Nic Lughadha, E.M.; Benardini, B. \& Chase, M.W. 2011. Phylogenetics, morphology, and evolution of the large genus Myrcia s.l. (Myrtaceae). International Journal of Plant Sciences 172: 915-934.
McVaugh, R. 1956. Tropical American Myrtaceae. Notes on generic concepts and descriptions of previously unrecognized species. Fieldiana, Botany 29: 145-228.

McVaugh, R. 1958. Myrtaceae (Calyptranthes and Marlierea). Memoirs of The New York Botanical Garden 10: 61-91.

McVaugh, R. 1968. The genera of American Myrtaceae - an interim report. Taxon 17: 354-418.

McVaugh, R. 1969. Myrtaceae. In: Maguire, B. and coll. (eds.), The Botany of the Guayana Highland. Part VIII. Memoirs of The New York Botanical Garden 18: 55-286.

Pires, J.M. \& Prance, G.T. 1985. The vegetation types on the Brazilian Amazon. In: Prance, G.T. \& Lovejoy, T.E. (eds.). Key environments: Amazônia. Pergamon, Oxford. Pp. 109-145.

Radford, A.E.; Dickson, W.C.; Massey, J.R. \& Bell, C.R. 1974. Vascular plant systematics. Harper \& Row, New York. 891p.

Rosário, A.S. \& Secco, R.S. 2006. Sinopse das espécies de Marlierea Cambess. (Myrtaceae) na Amazônia brasileira. Acta Amazonica 36: 37-52.

Rosário, A.S. 2012. Subtribo Myrciinae (Myrtaceae) na Amazônia brasileira, com ênfase no estado do Pará, Brasil. Tese de Doutorado. Escola Nacional de Botânica Tropical, Instituto de Pesquisas Jardim Botânico do Rio de Janeiro, Rio de Janeiro. 300p.

Sobral, M. 2003. A família das Myrtaceae no Rio Grande do Sul. Ed. Unisinos, São Leopoldo. 215p.

Sobral, M.; Proença, C.; Souza, M.; Mazine, F. \& Lucas, E. 2012. Myrtaceae. In: Lista de espécies da flora do Brasil. Jardim Botânico do Rio de Janeiro. $<$ http:// floradobrasil.jbrj.gov.br/2012/FB000171>. Acesso em 15 Ago 2012.

Thiers, B. [continuously updated]. Index Herbariorum: A global directory of public herbaria and associated staff. New York Botanical Garden's Virtual Herbarium. Disponível em $<$ http://sweetgum.nybg. org/ih/>. Acesso em 14 Ago 2012.

Wilson, P.G. 2011. Myrtaceae. In: Kubitzki, K. (ed.). Flowering plants. Eudicots: The families and genera of vascular plants. Vol. 10 Springer, Berlin, Heidelberg. Pp. 212-271.

\section{Lista de Exsicatas}

Berg, C.C.: 535 (1); Farney, C.: 1886 (6); Lindeman, J.C.: 292 (3); Maguire, B.: 35114 (2); Marinho, L.R.: 15 (5); Martinelli, G.: 14532 (6); Poole, J.M.: 2085 (1); Prance, G.T.: 20553 (3), 25536 (3); Sales, J.: 52 (2); Sandwith, N.Y.: 613 (3), 646 (1), 922 (3); Secco, R.: 642 (2). 